\title{
Unzuverlässiges Erzählen als Authentizitätsnachweis in einer unerfassbaren Welt: Beobachtungen zu Shida Bazyars Drei Kameradinnen
}

\author{
Eva Raschke (iD, Bremen \\ https://doi.org/10.37583/diyalog.1030306
}

\begin{abstract}
Deutsch)
Im vorliegenden Artikel soll nachgewiesen werden, dass die erzählerische Unzuverlässigkeit in Shida Bazyars Drei Kameradinnen passförmig ist zu einer im 21. Jahrhundert in Mitteleuropa diskursiv formulierten Unerfassbarkeit und Unfassbarkeit der Welt und des Selbst sowie zu einem Misstrauen gegenüber Objektivität und der Objektivier- und Vermittelbarkeit des eigenen Standpunkts.
\end{abstract}

Leitende These dabei ist, dass fantastische Exkurse, Selbstkorrekturen, Leerstellen, die Gleichzeitigkeit mehrerer Wahrheiten, Realitätsreferenzen und deren Brechung sowie Fiktionsbrechungen tragfester auf eine Realität referieren, die als unerfassbar gilt, als eine geschlossene Romanhandlung es hätte erreichen können.

Schlüsselwörter: Unzuverlässiges Erzählen, Literarische Authentizität, Intertextualität, Literarische Realitätsreferenzen, Postkoloniales Schreiben.

\section{Abstract (English)}

Unreliable Narration as Proof of Authenticity in an Incomprehensible World: Observations on Shida Bazyar's Drei Kameradinnen

This article aims to demonstrate that the narrative unreliability in Shida Bazyar's Drei Kameradinnen. It keeps an eye in the 21st century in Central Europe discursively and formulats incomprehensibility of the world and the self, a mistrust of objectivity, of the ability to objectify and to communicate the own point of view.

The leading thesis is that fantastic digressions, self-corrections, gaps, the simultaneity of several truths, references to reality and their refraction, as well as breaks in fiction, refer more solidly to a reality that is considered incomprehensible than a closed novel plot could have achieved.

Keywords: Unreliable Narrative, Literary Authenticity, Intertextuality, Literary Reality References, Postcolonial Writing. 


\section{EXTENDED ABSTRACT}

This article aims to demonstrate that the narrative unreliability in Shida Bazyar's Drei Kameradinnen is in keeping with an in the 21st century in Central Europe discursively formulated incomprehensibility of the world and the self, a mistrust of objectivity, of the ability to objectify and to communicate the own point of view. Within this contemporary discourse, it is aimed to show that Bazyar's novel can generate authenticity, credibility and a reference to the extra-literary world by a refusal of a chronological narrative as a world analogy, by the simultaneousness of several truths as a reference to intersectional polyphony as well as by broken references to reality as an approach to contemporary views on reality.

The leading thesis is that fantastic digressions, self-corrections, gaps, as well as breaks in fiction, refer more solidly to a reality that is considered incomprehensible than a closed novel plot could have done. The novel accompanies the reader through the slow-motion days of firstperson narrator Kasih and her friends Saya and Hani. From the first page the atmosphere is tense: A newspaper report preceding the text of the novel, in which a politically motivated attack by the protagonist Saya is reported, allows readers to look in flashbacks for the causes and motivation for the attack. While the time spent together by the three friends touches on daily global discourses about racism, privileges, gender relations, gentrification and poverty, the story jumps back to their childhood and youth.

The author repeatedly brings the "made-up" of the text into consciousness, demystifies and makes it impossible to sink into a continuous, coherent, chronologically or logically reliable novel. What is told is regularly corrected, what is marked as unexperienced is dreamed up in the subjunctive, questions are drafted and answers are withheld. By doing so, Bazyar's novel rejects the "classical" narrative tradition that considers chronological sequences, tension arcs or logical structures in the sense of life- course analogies.

As analytical tools that lend themselves to Bazyar's text, the means of post-colonial literary studies surprisingly prove to be fruitful: not because it was a post-colonial text, but because it was used to make written voices of the subaltern, polyphony and subversion writable. The analysis of inter-, meta, and intratextual references is an ideal for references to and breaks in reality. With an inter- or architextual view, references between several texts can be recognized. The reader is prevented from resolving the inconsistencies, leaving behind the irritation looking for what "really" was. Multi-perspective narration enables Bazyar to juxtapose and contrast the inner views of different figures, to allow criticism of one another and to reveal blind spots to one another. What one-character experiences and how a character evaluates and interprets it in a comprehensible way is contrasted with the experience and evaluation of other characters. There is no resolution, just as objective truth and knowledge are called into question in contemporary discourses.

By showing the figures in their knowledge as well as in their non-knowledge, the necessary limitation of one's own knowledge and reflection is illustrated. Furthermore, as the figures represent conflicting positions or their divergent perceptions are juxtaposed, the reader is required to make their own value judgment, their own weighing and positioning. Who is right? What is right? Where do I stand as a reader in this debate - and in current world events? Who defines what is right or false? Where are the limits? - The fact that the novel itself abstains from a final judgment in many places leaves room for resistance potential: In contradiction, in the simultaneous justification of two perceptions or perspectives, the friction arises that forces a step outside the novel and a connection to the world outside the novel. 
The text thus depicts the complexity, entanglement, intersectionality of social relationships and various levels of discrimination. Which of the characters has the right look at the observation? By not relieving the reader of answering this question, the text creates space for the ambivalences and ambiguities. It is also to add that different types of discrimination as a part of the intersectionality are nowadays of great importance. 


\section{Einleitung: Unzuverlässiges Erzählen als zeitgenössisches Erzählen}

„Unzuverlässiges Erzählen“ - was soll das sein? In dem Begriff sammeln sich diverse Rebellionen gegen Erzählkonventionen und Leseerwartungen, die uns im Lesen begegnen können: Die Absage an zeitliche oder räumliche Kontinuität, an erzählte Wahrheit, an Vertrauen in die Erzählstimme eines Romans und an das Wahren der Romanfiktion, an die unentlarvte Inszenierung des Schreibens. All diese Rebellionen oder Verweigerungen sind nicht neu. Sie wurden erprobt, theoretisch erfasst und beschrieben, in das Romangenre und seine Tradition inkludiert. Im vorliegenden Aufsatz soll am Beispiel von Shida Bazyars Drei Kameradinnen (Kiepenheuer \& Wisch 2021) nachgewiesen werden, wie die massive Kumulation dieser Ausbrüche sich ins Verhältnis setzen lässt zu einer aktuellen Weltlage, die geprägt ist von Pandemie, spürbarer Klimakatastrophe, nie dagewesenen globalen Flucht- und Migrationsbewegungen, technologischen Neuerungen, Abstiegserfahrungen einer Mittelschicht der Industrieländer und globalen Debatten um Diversität und strukturelle Diskriminierung.

Leitende These des vorliegenden Artikels ist dabei, dass die erzählerische Unzuverlässigkeit passförmig ist zu einer im 21. Jahrhundert in Mitteleuropa, in Deutschland, mehr als in den 70 Jahren zuvor erlebbaren Unerfassbarkeit und Unfassbarkeit der Welt, einer neuen Unplanbarkeit und einem Unvertrauen gegenüber der Erkennbarkeit der Welt, einem Misstrauen gegenüber Objektivität, gegenüber der Objektivier- und Vermittelbarkeit des eigenen Standpunkts und nicht zuletzt gegenüber (bspw. redaktionellen oder staatlichen) Autoritäten: Die Autorität des zuverlässigen Erzählens erscheint genauso bröckelnd wie die Autorität von staatlichen Institutionen und der nachrichtlichen Darstellung von Wahrheit und Erkenntnis. Innerhalb dieses zeitgenössischen Diskurses, so soll gezeigt werden, kann Bazyars Roman mehr Authentizität, Glaubwürdigkeit und letztlich Bezugnahme auf die außerliterarische Welt erzeugen, als es eine Zuverlässigkeit simulierende Inszenierung und eine kontinuierliche, abgeschlossene Romanhandlung hätten erreichen können.

\section{Drei Kameradinnen - thematische Annäherung an den Textinhalt}

Der Roman begleitet die Lesenden durch die in Zeitlupe durchlebten Tage von IchErzählerin Kasih und ihren Freundinnen Saya und Hani. Ab der ersten Seite ist die Atmosphäre zum Bersten gespannt: Eine dem Romantext vorangestellte Zeitungsmeldung, in der von einem politisch motivierten Brandanschlag durch die Protagonistin Saya berichtet wird, lässt die Lesenden in Rückblenden nach Ursachen und Motivation für diesen Anschlag suchen. Während die gemeinsam verbrachte Zeit der drei Freundinnen tagesaktuelle globale Diskurse um Rassismus, Privilegien, Geschlechterverhältnisse, Gentrifizierung und Armut streift, springt die Erzählung immer wieder zurück in die gemeinsame Kindheit und Jugend. Der gemeinsam erlebte Ausschluss (räumlich in einer Siedlung, sozial in der Stigmatisierung als „Migrantin“) bildet auch heute ein Band zwischen den jungen, urbanen Frauen, die, jede auf ihre Art, ihren Alltag und ihr Erwachsenwerden bewältigen: Kasih hat soeben ihr 
Soziologiestudium beendet und fühlt sich vom Jobcenter unter Druck gesetzt. Hani erledigt ihre Lohnarbeit bei einem vermeintlich sozial engagierten Projekt mit einem Übermaß an Konformität, Aufopferung und Leistungsbereitschaft. Saya hingegen beschäftigt sich vorrangig mit aktuellen politischen Ereignissen, wenn sie dem Gerichtsprozess gegen eine rechtsextreme Terrorgruppe, die gezielt Migrant/-innen ermordet hat, und den Leaks einer rechtsextremen Chatgruppe folgt. Anklänge an den „Nationalsozialistischen Untergrund“ (NSU) wie an publik gewordene rechtsextreme Chatgruppen innerhalb von deutscher Polizei und Bundeswehr drängen sich auf, werden aber auch immer wieder gebrochen. Saya diskutiert jederzeit, sogar mit Nazis, denen sie begegnet. Sie erträumt sich eine Welt ohne die Feindseligkeit, die ihren Alltag und den ihrer Freundinnen so massiv einschränkt und belastet.

Die drei erwachsen werdenden-gewordenen Freundinnen, die eigentlich nicht mehr in derselben Stadt leben, treffen anlässlich der Hochzeit einer vierten, Shaghayegh, zusammen, und verbringen die Tage und Stunden vor der Hochzeitsfeier gemeinsam. Während Lesende noch rätseln, was die Gewalttat von Saya letztendlich auslösen wird, laufen die Vorbereitungen für den Besuch der Hochzeitsfeier. Erzählerisch ineinander montiert gipfeln die Hochzeitsfeier und ein Großbrand gleichzeitig ineinander: eine mit Skepsis angenommene Einladung zu einem rauschenden Fest und die Todesangst einer Familie im brennenden Haus werden erzählerisch nebeneinander gestellt, bis die Erzählerin auf den letzten Seiten Tat, Täter/innen und nicht zuletzt die Leser/-innen selbst entlarvt.

\section{Die Welt im Werk und das Werk (positioniert) in der Welt: Romanhandlung und Realitätsbezug}

Die Autorin rückt das „Gemachtsein“ (Schahadat 1995: 374) des Textes immer wieder ins Bewusstsein, entzaubert, verunmöglicht ein Versinken in einer kontinuierlichen, schlüssigen, zeitlich oder logisch zuverlässigen Romanhandlung. Regelmäßig wird Erzähltes korrigiert, wird als nicht-erlebt Markiertes im Konjunktiv erträumt, werden Fragen aufgeworfen und Antworten vorenthalten. Mit einem Repertoire an erzählerischen Mitteln verlangt die Autorin den Lesenden ab, sich auf absolute Unzuverlässigkeit einzulassen. Gleichzeitig gewinnt die Romanhandlung so an Realitätsbezug: Als eben nicht einzigartiges, nur einmal geschehenes Erlebnis wird ein Spektrum an Alltag und Lebenserfahrung erahnbar, das sich mit definitiven, gesicherten Aussagen nicht oder nur stereotyp hätte abbilden lassen. Gerade weil der Roman Vorurteile und Klischees thematisiert, funktioniert er umso glaubwürdiger als zeithistorisch positionierter Text, je weniger erwartbar die Romanhandlung verläuft.

Als analytisches Werkzeug, das sich für Bazyars Text anbietet, erweist sich überraschend die postkoloniale Methode (mit dem von Albrecht formulierten wichtigen Hinweis, „Autorinnen und Autoren nicht auf ihre Herkunft und auf dieser Herkunft vermeintlich entsprechende Themen festzulegen“ (Albrecht 2005: 252) und unter Berücksichtigung der von Günther formulierten Kritik (vgl. Günther 2002: 153)) als fruchtbar: Nicht, weil es sich um einen im klassischen Sinne postkolonialen Text 
handelte, sondern weil so die im Roman exponiert formulierten Stimmen der Subalterne, ihre Vielstimmigkeit und Subversion, beschreibbar gemacht werden. Für die Realitätsbezüge und -brechungen bietet sich die Analyse von inter-, meta- und intratextuellen Bezügen an, weil mit ihrer Hilfe Texträume, Dialogizität und überschrittene Textgrenzen erfasst werden können.

\subsection{Die Verweigerung einer chronologischen Erzählung als Weltanalogie}

Bazyars Roman verweigert sich „klassischen“ Erzähltraditionen, die zeitliche Abfolgen, Spannungsbogen oder logischen Aufbau im Sinne von Lebensverlaufsanalogien berücksichtigen. Bemerkenswert ist diese Verweigerung nicht nur, weil fraglich ist, ob die beschriebene Erzähltradition heute überhaupt noch hegemonial ist, sondern vielmehr in ihrer abwertenden Markierung als Erzählform der „Deutschlehrer und Deutschlehrerkinder" (Bazyar 2021: 63), wird doch so die Ablehnung ausgeweitet auf eine vermeintlich milieu- bzw. schichtspezifische Erzählhaltung und damit ein soziales Distinktionsmittel. In der Rebellion gegen einen zeitlichen Kontinuitätsanspruch liegt zugleich die Rebellion gegen ebendiese bildungsbürgerliche Form, die nicht nur einer Erzählung, sondern analog auch einer extra-literarischen Lebensplanung, logischen Aufbau und Zielstrebigkeit abverlangt. Interessanterweise wurde genau diese Weigerung, den „Vorstellungen von Linearität und Fortschritt“ zu genügen, andernorts als postkoloniales Mittel beschrieben (vgl. Schößler 2006: 150).

Auffällig und vielleicht genretypisch für eine Coming-of-Age-Erzählung ist das Fehlen einer Perspektive auf die Zukunft im Roman: Die Gedankenwelt der Erzählerin und der Protagonistinnen ist einzig auf Vergangenheit und Gegenwart ausgerichtet. Pläne, Perspektiven, Wünsche oder Träume für die nähere oder fernere Zukunft tauchen weder konkret noch abstrakt auf. Die Verweigerung eines Happy Ends, eines greifbaren Endes überhaupt, bildet eine Weltanalogie, in der weniger denn je abgesehen werden kann, was die Zukunft bringt. Die gesamtgesellschaftliche, generationelle Erfahrung in Post-Wachstums-Ära und Pandemie, dass das Leben nur noch sehr bedingt vorausgeplant, Biografien nur selten wie vorgezeichnet gestaltet werden können, bildet sich in der Auslassung einer Zukunftsperspektive genauso wie in der Rebellion gegen die Erzählform vermeintlicher „Deutschlehrerkinder“ im Roman ab.

\subsection{Fantastische Exkurse, Selbstkorrekturen und Leerstellen als Authentizitäts- merkmale}

Jenseits der Kennzeichnung von Drei Kameradinnen auf den Umschlagsseiten als „Roman“, also als einem fiktiven, inszenierenden Genre zugehörig, nutzt Bazyars Text verschiedene Mittel, um sich ins Verhältnis zu einer außertextlichen Realität zu setzen. Dieses Verhältnis ist weniger fiktiv und verspielt als es zunächst scheinen mag. Mehr noch: Gerade diese Mittel, so soll im Folgenden gezeigt werden, bilden ein starkes Band zu zeitgenössischer Realität und Realitätsdiskursen. 
So wird die soziale Positionierung der drei Freundinnen durch eine Rückblende zu ihrer Schulabschlussfeier, dem „Abiball“, illustriert: Wie ihre Eltern die Bedeutung der Inszenierung entweder nicht ermessen können oder - aus hegemonialer Sicht vollkommen „unangemessen“ bewerten, mögen sich auch die drei Abiturientinnen Saya, Hani und Kasih nicht recht in die durchchoreografierten Abläufe einfügen (vgl. Bazyar 2021: 223f.). Beim späteren Abiball des Bruders hingegen, berichtet der Roman, hätten Kasih und ihre Eltern mehr Vorwissen zu hegemonialen Gepflogenheiten und Ritualen gehabt.

Beim Abiball meines Bruders habe ich es anders gemacht. Ich habe meinen Eltern gesagt, dass man dahin muss. [...] Ich habe meinem Bruder gesagt, dass er sich einen Anzug kaufen und zum Friseur gehen muss. Ich saß mit meinen Eltern in den Reihen der Familien und mein Bruder ganz vorne, in den Reihen der Abiturienten. Als man die Namen der Abiturienten aufrief, wurde sein Name, unser Name, erwartungsgemäß falsch ausgesprochen. [...] Merkwürdig war auch, den anderen stolzen Eltern dabei zuzuschauen, wie sie ihre Kinder beklatschten, während der eigene Bruder wohl nur aus Zufall sein Abitur gemacht hat. (ebd.: 226f.)

Eine Integration, eine Anpassung in die vorgefundenen Rollenerwartungen und hegemonialen kulturellen Wertmuster lässt das Unbehagen der lesenden Beobachterin der Szenerie kurzzeitig in Erleichterung über die gelungene Verständigung, die Möglichkeit einer Gemeinsamkeit bei genügender Anstrengung, wenn auch auf Kosten der „Integrierten“ (beispielsweise durch einen falsch ausgesprochenen Namen), weichen. Doch sofort wird diese wieder genommen:

Das da oben habe ich zugegebenermaßen gerade erfunden. Mein Bruder hat gar kein Abi. Ich denke aber, wenn er Abi gemacht hätte, wäre es doch sicher genau so gelaufen. Ich habe allerdings Zweifel daran, dass meine Eltern wirklich mitgekommen wären. [...] Ich will jetzt aber noch weiter erfinden, dass dann der Erdkundelehrer meines Bruders, der auch mal mein Erdkundelehrer war, am Büfett hinter meinem Vater stand und dachte, das sei ein toller Anlass, ihn ein wenig kennenzulernen, während mein Vater fand, das alles sei eher so ein Anlass, um mindestens so viel zu essen, wie man bezahlt hatte. (ebd.: 228)

Es gab nie einen solchen Abiball des Bruders, der die Scham und das „NichtPassen“ von Kasihs Abiball hätte wiedergutmachen können. Desillusionierung, Enttäuschung macht sich breit: Für den verpatzten Abiball Kasihs gibt es keine zweite Chance; die Genugtuung durch eine vermeintlich erfolgreiche wie fragwürdige (da sich schlicht der hegemonialen Erzählung unterwerfenden) Integrationserzählung bleibt verwehrt. Die Romaninszenierung spiegelt den deutschen Integrationsdiskurs und eine Integrationsrealität, in der ein nominell erwünschtes gesellschaftliches Aufsteigen de facto rassistisch und schichtspezifisch verschlossenen Bildungswegen gegenübersteht. Bazyar lässt Verschiedenheit in gegenseitigem Unverständnis stehen, erlaubt Reibung und Widerborstigkeit in ihrem Text. Sie unterwirft an dieser Stelle den erzählten Vater nicht der kolonialen Erzählung des tumben, unverständigen Migrierten, sondern gibt ihm die Souveränität, beobachten und teilnehmen $\mathrm{zu}$ können und trotzdem nicht einverstanden zu sein. Homi Bhabha hat dieses in Bazyars Roman beobachtbare Phänomen bereits als „Aushandeln der Andersheit“ (De Toro 2002: 22) formuliert. Auch seine Begriffe „des 'unhomely", des ,inbeetween' und der ,mimicry" (zitiert nach 
De Toro, ebd., Hervorhebungen im Original) beschreiben das, was der Roman an dieser Stelle in Szene setzt, treffend.

Ein weiterer Kunstgriff, der die Romanhandlung als inszeniert und fiktiv entlarvt, ist das explizite Aufwerfen von Leerstellen:

\begin{abstract}
Jetzt freut ihr euch vermutlich, denn wenn es um Hanis Mutter geht, erzähle ich vielleicht ganz nebenbei, welcher „ethnischen Gruppierung” sie eigentlich angehört und warum diese der Grund war, dass ihre Familie fliehen musste und woher eigentlich genau, in welchem Jahr, damit ihr das abgleichen könnt mit den Sachen, die ihr damals so schrecklich betroffen in den Nachrichten verfolgt habt, und den richtigen WikipediaArtikel dazu öffnen könnt, um sicherzugehen, nichts durcheinanderzubringen. Das alles erzähle ich euch jetzt aber natürlich nicht, ihr erfahrt nur das, was man über Hanis Mutter wissen muss, damit man sie als Heldin auszeichnen und im Abspann nennen kann. (Bazyar 2021: 280f.)
\end{abstract}

Statt schlicht nicht $\mathrm{zu}$ benennen, $\mathrm{zu}$ übergehen, positioniert sich der Roman im zeitgenössischen „Textraum, in dem Sinn durch das Aufeinandertreffen verschiedener Texte und Kontexte generiert oder auch unterlaufen wird“" (Schahadat 1995: 367). Mehr noch, die exponierte Verweigerung einer Orientierung in der außerliterarischen Welt, gar das Lächerlichmachen einer solchen, sind selbst ein intertextueller Bezug auf historische, allgemein bekannte Ereignisse wie Krieg, Flucht und Emigration nach Deutschland. Damit wendet die Autorin Strategien an, die in der germanistischen Forschung als ,postkolonial” beschrieben wurden (vgl. Dürbeck 2014: 30f.): Durch die Sichtbarmachung und Nachahmung von „Machtstrategien“ (ebd.), beispielsweise Zahlen-/Faktenwissen höher zu bewerten als Erfahrungswissen, Rationalität Emotionalität scharf abgegrenzt gegenüberzustellen oder Realität und Imagination als Gegensätze $\mathrm{zu}$ verhandeln und $\mathrm{zu}$ bewerten, macht sich der Text der kolonialen Überprüfung widerspenstig: Auf Wikipedia über Krieg und Flucht nachlesen muss an dieser Stelle nur, wer diese nicht selbst erlebt hat, und deshalb keinen emotionalen, sondern einen an äußeren Fakten orientierten, Zugang zu diesen Themen hat. Durch eine Kenntnis der Sachlage aber zu glauben, auch die betroffenen Personen verstehen und ihre gesellschaftliche Position bewerten zu dürfen, verbietet der Roman mittels dieser Entlarvung der Lesenden. Analytisch beschrieben wurde ein passförmiger Textzugang bereits von intertextuellen Ansätzen:

[...] sowohl lector als auch scriptor werden in einem Gedächtnisraum angesiedelt, in dem der Text selbst zum Mittelpunkt wird und an dem Autorin und Leserin gleichermaßen teilhaben. Die Autorin schreibt Spuren des kulturellen Gedächtnisses (bewußt oder unbewußt) in den Text ein, die Leserin wiederum wendet, je nach kulturellem Hintergrund, Wissensstand oder Intention, eigene Lesestrategien an. (Schahadat 1995: 371)

Lesende können an dieser Stelle praktisch erfahren, was Schahadat theoretisch skizziert: „eine schrittweise ablaufende Lektüre [...]“ (ebd.: 376), bestehend aus „Erkennen der Allusion - Identifikation des Prätextes - Modifikation des Oberflächensinns der Allusion - Aktivierung des Prätextes" (ebd.).

So rabiat und rücksichtslos, falls eine Romanerzählung dies sein kann, wie kurz vor dem Gipfelpunkt der Romanhandlung, sind die Selbstkorrekturen und 
Selbstentlarvungen als Fiktion selten, wie in dem Moment, in dem die Protagonistin Hani retrospektiv aus der Romanhandlung gestrichen wird:

\begin{abstract}
Hani hat in ihrem eigenen Bett geschlafen, wenn ihr euch richtig erinnert, damit sie in Ruhe duschen und mit ihrem Fahrrad ins Büro fahren kann. Nur habe ich euch verschwiegen, dass Hanis Bett nicht in einer Wohnung im Stadtteil nebenan steht, sondern in einem anderen Land. Genau wie ihr Fahrrad und ihr Büro und ihr Pflaumentee und ihre Chefin und die Kollegen, die sie und ihre Sorgfalt und Freundlichkeit ausnutzen. Als der blutrünstige, schonungslose Krieg in Hanis einstiger Heimat für beendet erklärt worden war, schickte man Leute wie sie und ihre Familie zurück, ganz gleich, wie gut ihre Noten waren. Habt ihr ernsthaft geglaubt, jemand hätte sich jemals für Hanis gute Noten interessiert? (Bazyar 2021: 348)
\end{abstract}

Die bundesrepublikanische Realität von Abschiebungen, nur zu oft gegen jedes humanistische oder gar wirtschaftslogische Wissen und Gefühl, wird so in den Romanalltag geholt. Mehr noch als dass er eine Hauptfigur rückwirkend aus der Handlung streicht und in die Fantasie und die Erinnerung der Protagonistin verbannt, macht diese schnöde Streichung Platz für die Trauer um und die Sehnsucht nach den Personen, die aufgrund einer rigorosen deutschen Asylpolitik nicht (mehr) hier sind. Ausgerechnet Hani, die Fleißige, Aufsteigerin, die Assimilierte (vgl. ebd.: 121), Leistungsbereite ist nun gleichzeitig unauslöschbar in die Romanhandlung verstrickt und ungreifbar abwesend. Die Lesenden bleiben zurück als Betrogene: Sie haben sich auf die erzählte Hani eingelassen, sind mit ihr durch die deutsche Großstadt geradelt und haben sie als mit Kolleg/-innen in einem deutschen Hipster-Büro hadernd imaginiert. Wieder mit Mitteln der postkolonialen Ästhetik, nämlich mit der „spielerischen oder kritisch-subversiven Sichtbarmachung der Diskursmuster und Dichotomien, die die symbolische Ordnung [...] strukturieren“ (Uerlings 2006: 3), mutet die Autorin durch eben diesen Betrug den Lesenden das Nachspüren des eigentlichen Betrugs zu: Der Lüge von (schulischer) Leistung, die sich lohne oder der Lüge davon, dass erfolgreiche Assimilation eine hinreichende Begründung für einen sicheren Aufenthaltsstatus sein könne. Schließlich, schmerzlich, dokumentiert sie auch die um eine lebenslange Freundinnenschaft betrogenen Protagonistinnen, eine Freundinnenschaft, die ohne Staatsgrenzen, Pässe und deutsche Abschiebegesetze hätte entstehen können. Anklänge an das, was Bhabha als Ironie als Mittel im Verhältnis von postkolonialer und kolonialer Literatur beschrieben hat (vgl. Lützeler 2005: 18), werden hier evoziert - wenn auch an dieser Stelle mit zu bitterem Beigeschmack, um wirklich Ironie und nicht Sarkasmus zu sein.

Besonders auffällig sind die Widersprüche im Erzählten zwischen „Zeitungsbericht" am Romaneingang und Romanhandlung am Ende.

\footnotetext{
Kurz darauf attackiert sie vor einem Café in der Bornemannstraße einen Mann unter ,Allahu Akbar'-Rufen. Volker M. befindet sich in ärztlicher Behandlung. Über seinen Anwalt lässt er mitteilen: „Wir waren lange genug tolerant. Es sind Menschen wie Saya M., die die Sicherheit unseres Landes gefährden. Wie viele Anschläge muss es denn noch geben?“" (Bazyar 2021: 4f.)
}

Der reißerische Zeitungsartikel kollidiert auf besondere Weise mit der Romanerzählung. Hier attackiert Saya keinen attributlosen „Mann“, sondern diskutiert mit einem 
„Paradenazi“ (ebd.: 339) namens „Patrick Wagenberg“ (ebd.: 155, 338), der online wie offline rechtsextreme Propaganda betreibt. Saya entlarvt seine „Meinung“ als Hass, der auf die Vernichtung jener Menschen abzielt, die nicht in das Weltbild des Nazis passen (vgl. ebd.: 341). Dass im Zeitungsbericht „Volker M.“ und in der Romanhandlung „Patrick Wagenberg“ auf Saya treffen - Ausdruck einer Medienkritik? Einer schlechten journalistischen Recherche? Einer politisch positionierten Presse, die dem vermeintlich „geschädigten Deutschen“ einen noch „deutscheren“, einen sprechenden Namen verpasst: „Volker"? Oder vielmehr ein Indiz dafür, dass Saya den Falschen wiederzuerkennen glaubt, aber dass gleichzeitig der „Falsche“ der Richtige ist - dass Saya also, indem sie ihr Gegenüber verkannte (nämlich die Person verwechselt hat), ihn doch gerade umso schärfer erkannt hat (nämlich als Nazi, ein Bruder im Geiste des Patrick Wagenberg)?

Die Auflösung der Ungereimtheiten bleibt den Lesenden verwehrt, zurück bleibt die Irritation, die danach sucht, was nun ,wirklich“ war - auch danach, ob Saya im Recht war: Ob Zeitungsartikel oder Romanerzählung glaubwürdiger sind, oder ob sie vielleicht beide gleich unglaubwürdig sind, jeweils als entkontextualisierte bzw. in neuen Kontext gezerrte und dadurch verzerrte Ausschnitte, die aber als solche kein Urteil von Außenstehenden mehr erlauben. Mit einem inter- oder architextuellen Blick lassen sich hier ,Text-Text-Bezüge“ erkennen und ,[...] an Stelle des Einzeltextes tritt ein Textraum, in dem Sinn durch das Aufeinandertreffen verschiedener Texte und Kontexte generiert oder auch unterlaufen wird" (Schahadat 1995: 367). Schahadat beschreibt dieses Aufeinandertreffen auch als eine „Konfrontation“, innerhalb derer „die Differenzen zwischen den Texten“ eine Spannung erzeugten, „die die semantische Bewegung des Intertextes“ ausmachten (ebd.: 373). Genau diese Bewegung, die auf der rezeptiven Seite zu verorten ist, wird auf die Spitze getrieben, wenn der Text sich selbst als eine „Version“ benennt, eine subjektive und beschnittene Auswahl an Informationen:

\begin{abstract}
Ich war außerdem sauer, weil ich mir Wagenbergs Dünnpfiff durchlesen musste. Es tut mir leid, dass ihr euch das jetzt auch durchlesen musstet. Weil ihr aber das Internet schon mal benutzt habt, war das ja wohl nicht das erste Mal, dass ihr so was gelesen habt. Falls doch, muss ich euch noch weiter beunruhigen, denn das hier waren nur meine abgeschwächten Formulierungen, die echte Nachricht war schlimmer. Das ist wie mit dem Artikel ganz am Anfang, wisst ihr noch? In dem steht, Saya hätte vor dem Brand „Allahu Akbar“ gerufen und so, der war in echt auch viel schlimmer, seid froh, dass ihr nur meine Version kennt. (Bazyar 2021: 257)
\end{abstract}

Der Text verweigert sich auch an dieser Stelle einer Erzähltradition, die Konsistenz und Zuverlässigkeit in der Romaninszenierung verlangt. Und während der Text insgesamt tatsächlich klassische Romanelemente wie Erzählstimme, Rückblenden, abschließend erzählte Episoden, benannte und kontinuierliche Hauptfiguren u. v. m. aufgreift, bricht er an dieser Stelle abrupt mit der Tradition. Stattdessen: „Parodie, Kontradiktio, Kontrafaktur” als „ein Akt des Gegen- und Neuschreibens“ (Schahadat 1995: 375). 


\title{
3.3 Gleichzeitigkeit mehrerer Wahrheiten als Referenz auf intersektionale Vielstimmigkeit
}

Multiperspektivisches Erzählen ermöglicht es Bazyar, die Innensicht verschiedener Figuren nebeneinander und gegeneinander zu stellen, aneinander Kritik üben zu lassen und einander blinde Flecken aufzuzeigen. Was die eine Romanfigur erlebt und wie sie es nachvollziehbar bewertet und deutet, wird kontrastiert mit dem Erleben und Bewerten anderer Figuren. Eine Auflösung unterbleibt, wie auch in zeitgenössischen Diskursen objektive Wahrheit und Erkenntnis in Frage gestellt werden. Nicht nur durch Diskussionen über Privates und Politisches unter Saya, Hani und Kasih (bspw. Bazyar 2021: 62f.), in denen sie unterschiedliche Argumentationsstränge verhandeln, werden verschiedene Perspektiven gegenübergestellt. Auch die Innensicht von Randfiguren wird aufgegriffen, um deren Deutungsweisen abzubilden und Ambiguitäten Raum zu geben.

So montiert die Erzählung das Sehen einer Nachrichtensendung im Fernsehen durch Saya neben das Sehen derselben Sendung durch eine unbekannte Hausnachbarin:

\begin{abstract}
Die Nachrichtensprecherin begrüßte Saya und Saya öffnete kurz die Augen, um zu sehen, wie die Sprecherin aussah. Weiß. Das hatte sie sich gedacht. [...] Ein letzter Blick und dann ab ins Niemandsland, dachte sie und machte den dritten Haken: weiß. Check. Drei Frauen in fünf Minuten aus genau einer Gruppe: der privilegierten. [...] In der Wohnung über ihr saß währenddessen eine Frau vor dem Fernseher, die gerade ihre Steuererklärung machte und mit halbem Ohr die Sendung verfolgte. [...]Was für eine tolle Quote, dachte sie. Drei Personen innerhalb von fünf Minuten an so prominenter Stelle aus genau einer Gruppe: der benachteiligten. In den Nachrichtensendungen ihrer Kindheit hatten Frauen maximal das Wetter angesagt. Heute waren sie viel präsenter. Saya in der Wohnung darunter dachte gar nicht daran, sich darüber zu freuen, denn sie hatte dem Fernseher ja den Rücken zugekehrt, das Thema war für sie gegessen. (Bazyar 2021: 231ff.)
\end{abstract}

Damit bildet der Text die Vielschichtigkeit, Verschränkung, die Intersektionalität sozialer Beziehungen und verschiedener Diskriminierungsebenen ab. Welche der beiden Figuren hat den richtigen Blick auf die Beobachtung? Indem der Text den Lesenden die Beantwortung dieser Frage nicht abnimmt, entwickelt er eine „Poetik der Ambivalenz, die Subversion und Affirmation verknüpft“ (Schößler 2006: 148). Saya, in einer erzählerischen Rückblende zu einer Familienfeier ihres Weißen ${ }^{1}$ Partners Leo eingeladen (vgl. Bazyar 2021: 168) muss erleben, wie eine andere Frau, die, wie Saya, neben Leo sitzt, für dessen Partnerin gehalten wird. Diese Frau, Lena genannt, ist Weiß - und in diesem Vorfall zeigt sich für Saya eine Erniedrigung, ein durch und durch rassistischer Verweis auf ihren sozialen Platz, der in den Augen der Sprecherin nicht an der Seite eines Weißen Mannes sein kann. Dieses Erlebnis, das als für Saya sehr bedeutsam und lange nachwirkend erzählt wird, wird im Romanverlauf mit einer gänzlich anderen Perspektive kontrastiert:

\footnotetext{
${ }^{1}$ Um zu verdeutlichen, dass es sich bei „Weiß“ und „Weißsein“ nicht um biologische Eigenschaften oder reelle Hautfarben handelt, sondern um politische und soziale Konstruktionen, werden diese Begriffe groß geschrieben.
} 
Nach all den Malen, die ich diese Geschichte hören musste, ahne ich erst jetzt, dass Lena, die in dieser Story nicht besonders gut wegkommt und noch nicht einmal einen echten Namen hat, die Geschichte sicher auch nicht vergessen hat. Dass Lena im Nachhinein ebenfalls mit Leuten sprach, um sich bestätigen zu lassen, dass sie sich völlig zu recht darüber geärgert hat, für Leos Freundin gehalten zu werden, obwohl die doch genauso neben Alice, Leos Schwester, saß. Ich würde jetzt gerne Saya von diesem viel zu spät gefallenen Groschen erzählen, damit wir und beide schämen können, Saya noch ein bisschen mehr als ich. Um uns erst für unsere Ignoranz zu schämen, und dann wie unsensible Trampel fühlen und uns schließlich dafür zu schämen, die unsensiblen Trampel zu sein, die wir selbst so verachten. (Bazyar 2021: 171f.)

In dieser „Stimmenvielfalt“ (Uerlings 2005: 32) und in den „Selbstdistanzierungen“ (Lubrich 2005: 38, Anm. 161), denen Bazyar an dieser Stelle Raum gibt, lässt sich postkoloniales Potenzial im Sinne von Widerständigkeit und Nicht-Passförmigkeit entdecken: Homophobie und Heteronormativität werden eingestanden, eigene Ignoranz und damit auch eigene Privilegien thematisiert, nämlich: nicht zentrale Zielscheibe von homophoben Weltbildern, An- und Übergriffen zu sein. Die Gleichzeitigkeit mehrerer Wahrnehmungen und damit auch mehrerer Wahrheiten wird in dieser erzählerischen Inszenierung wie unter einem Brennglas zugespitzt und kontrastiert. Berechtigung wird beiden zugestanden, auch wenn die Protagonistinnen der Erinnerung, Lena und Saya, getrennt voneinander und damit ohne Verständigung oder gar Solidarisierung für einander verbleiben.

In diesem Erzählabschnitt demonstriert der Text nicht nur „Selbstreflexivität“ (Dürbeck 2014: 20), sondern erzeugt auch eine „Polyphonie und Dezentrierung in den Schreibweisen“ (ebd.), wie es sonst für postkoloniales Schreiben beschrieben wurde. Das formulierte „Ahnen“ zeigt Anklänge an die von Dürbeck formulierte „Bedeutung des Imaginären“ (ebd.: 19) in postkolonialen Ansätzen: Die beschriebene Stimmenvielfalt beruht hier nicht auf gesichertem Wissen und interpersonalem Austausch, sondern rein auf der Fantasie der Ich-Erzählerin Kasih. Eben diese Polyphonie und Imagination bietet Lesenden sehr offen an, sich zum Text ins Verhältnis zu setzen - und ebenso den Text ins Verhältnis zu aktueller Weltlage und globalen Diskursen zu setzen. Nicht nur Handlung und Erzähltes, sondern darüber hinaus die Schreibweise selbst stellt hier einen authentischen Bezug zur außerliterarischen Welt her.

\subsection{Gebrochene Realitätsreferenzen als Annäherung an die Realität}

Bazyars besonderer Erzählstil zeigt sich sowohl in Realitätsreferenzen und deren Brechung als auch in Fiktionsbrechungen, wenn die Romanhandlung unterbrochen wird, um die Lesenden direkt anzusprechen, Hypothesen über sie zu formulieren und ihnen Fragen zu stellen.

Einen Haupterzählstrang bildet der Gerichtsprozess gegen eine rechtsextreme Terrorgruppe, die gezielt Migrant/-innen ermordete. Die zahlreichen Analogien („Untergrund“ (Bazyar 2021: 83), „Selbstenttarnung“ (ebd.: 84), „V-Mann, der nachweislich bei einem der Morde dabei war“ (ebd.) legen Assoziationen an den 
„Nationalsozialistischen Untergrund“ (NSU) nahe. Doch werden diese Analogien wieder gebrochen: Der NSU hat beispielsweise nicht ,vorzugsweise muslimische weibliche Menschen“ (Bazyar 2021: 83) ermordet, sondern in der Mehrzahl Männer. Während im Roman einer „der Angeklagten [...] so jung“ (ebd.: 298) genannt wird, war im NSU-Prozess keine/-r der fünf Angeklagten unter 35 Jahren alt. Warum dieser starke Realitätsbezug, der dann irritiert wird? In der Nicht-Passförmigkeit, in der Abweichung zeigt sich, dass der NSU, der Terror gegen (vermeintliche) Immigrant/-innen, keine Einzigartigkeit in sich trägt. In der Verfremdung des Einzelphänomens wird die Struktur sichtbar: Die Vielzahl der rassistisch motivierten Gewalttaten in Deutschland, die Vielzahl der rechtsterroristischen Organisierungen, die Potenzialität der Wiederholung ihrer Taten. Gleichzeitig: Weil die Dimensionen des realen NSU im Roman nie abschließend oder gar angemessen erfasst, abgebildet oder kommentiert werden könnten, erlaubt die fiktionale Variante einen Umgang, eine Erfassbarkeit, eine Positionierung.

\section{Fazit}

Im vorliegenden Artikel wurde anhand von Shida Bazyars Drei Kameradinnen die These untersucht, ob erzählerische Unzuverlässigkeit passförmig ist zu einem spezifischen zeitgenössischen Zeitgeist in Mitteleuropa. Mithilfe von intertextuellen und postkolonialen literaturwissenschaftlichen Bezugnahmen konnte gezeigt werden, dass unzuverlässiges Erzählen in einer Welt der unerfassbaren Informationslagen und nie abgeschlossenen Positionierungen in ihr als Authentizitätsnachweis wirkt. Was paradox klingt, konnte im Konkreten nachgewiesen werden: Der literarische Text wirkt erst über sich hinaus in den außerliterarischen Raum durch seine Polyphonie, seine Widersprüchlichkeit, seine Hermetik. Die Brüche und Unvollständigkeiten referieren tragfester auf eine Realität, die als unüberschaubar gilt und in der abschließende (Selbst)Erkenntnis unmöglich scheint, als eine auktorial geleitete Erzählung es vermocht hätte.

Indem die Figuren in ihrem Wissen wie in ihrem Nicht-Wissen gezeigt werden, wird die notwendige Begrenztheit eigener Erkenntnis und Reflexion illustriert. Und indem die Figuren widerstreitende Positionen vertreten oder ihre abweichenden Wahrnehmungen nebeneinandergestellt werden, wird den Lesenden ein eigenes Werturteil, eine eigene Abwägung und Positionierung abverlangt. Wer hat Recht? Was ist richtig? Wo stehe ich als Lesende/-r in dieser Debatte - und im aktuellen Weltgeschehen? - Dass sich der Roman selbst vielerorts eines abschließenden Urteils enthält, lässt Raum für widerständiges Potenzial: Im Widerspruch, in der gleichzeitigen Berechtigung zweier Wahrnehmungen oder Sichtweisen, entsteht die Reibung, die ein Hinaustreten aus dem Roman und eine Verknüpfung zur Welt außerhalb des Romans erzwingt. Die Analyse ausgewählter Romanausschnitte konnte zeigen, dass diese Vielstimmigkeit zu einem widerborstigen Chor wird, der nicht einig sein kann und es auch nicht muss. Ebenso, wie die Realitätsbezüge aufdringlich hergestellt und dann wieder irritiert werden, werden auch fantastische Exkurse, Selbstkorrekturen und 
Leerstellen als Mittel genutzt, die außerliterarische Welt abzubilden, zu kommentieren und zu remodellieren. Und wo die Realität in ihrer Wucht oder in ihrer Verstrickung hermetisch bleibt, nähert sich der Romantext fragmenthaft, dadurch jedoch nicht weniger stark.

\section{Literaturverzeichnis}

Albrecht, Monika (2005): Gegenwartsliteratur aus postkolonialer Sicht. Michael Krüger: Himmelfarb und Jeannette Lander: Jahrhundert der Herren. In: Dunker, Axel (Hrsg.): (Post-)Kolonialismus und Deutsche Literatur. Impulse der angloamerikanischen Literatur- und Kulturtheorie. Bielefeld: Aisthesis, 251-265.

Albrecht, Monika (2008): „Europa ist nicht die Welt“. (Post)Kolonialismus in Literatur und Geschichte der westdeutschen Nachkriegszeit. Bielefeld: Aisthesis.

Bazyar, Shida (2021): Drei Kameradinnen. Köln: Kiepenheuer \& Witsch.

De Toro, Alfonso (2002): Jenseits von Postmoderne und Postkolonialität. Materialien zu einem Modell der Hybridität und des Körpers als transrelationalem, transversalem und transmedialem Wissenschaftskonzept. In: Hamann, Christof / Sieber, Cornelia (Hrsg.): Räume der Hybridität. Postkoloniale Konzepte in Theorie und Literatur. Hildesheim u.a.: Georg Olms Verlag, 15-52.

Dürbeck, Gabriele (2014): PostkolonialeStudien in der Germanistik. Gegenstände, Positionen, Perspektiven. In: Dürbeck, Gabriele / Dunker, Axel (Hrsg.): Postkoloniale Germanistik. Bestandsaufnahme, theoretische Perspektiven, Lektüren. Postkoloniale Studien in der Germanistik Band 5. Bielefeld: Aisthesis, 19-70.

Günther, Petra (2002): Die Kolonisierung der Migrantenliteratur. In: Hamann, Christof / Sieber, Cornelia (Hrsg.): Räume der Hybridität. Postkoloniale Konzepte in Theorie und Literatur. Hildesheim u.a.: Georg Olms Verlag, 151-159.

Lubrich, Oliver (2005): Welche Rolle spielt der literarische Text im postkolonialen Diskurs? In: Archiv für das Studium der neueren Sprachen und Literaturen 157 (242). Bamberg: Erich Schmidt Verlag, 16-39.

Lützeler, Paul Michael (2005): Postmoderne und postkoloniale deutschsprachige Literatur. Diskurs Analyse-Kritik. Bielefeld: Aisthesis.

Schahadat, Schamma (1995): Intertextualität. Lektüre - Text - Intertext. In: Pechlivanos, Miltos u.a. (Hrsg.): Einführung in die Literaturwissenschaft. Stuttgart u.a.: Metzler, 366-377.

Schößler, Franziska (2006): Literaturwissenschaft als Kulturwissenschaft. Eine Einführung. Tübingen: UTB.

Uerlings, Herbert (2005): Kolonialer Diskurs und Deutsche Literatur. Perspektiven und Probleme. In: Dunker, Axel (Hrsg.): (Post-)Kolonialismus und Deutsche Literatur. Impulse der angloamerikanischen Literatur- und Kulturtheorie. Bielefeld: Aisthesis, 17-44.

Uerlings, Herbert (2006): „Ich bin von niedriger Rasse“. (Post-)Kolonialismus und Geschlechterdifferenz in der deutschen Literatur. Köln u.a.: Böhlau. 